\title{
Optic nerve sheath diameter change in prediction of malignant cerebral edema in ischemic stroke: an observational study
}

Seong-Joon Lee ${ }^{1}$, Mun Hee Choi', Sung Eun Lee ${ }^{1}$, Ji Hyun Park ${ }^{2}$, Bumhee Park ${ }^{2,3}$, Jin Soo Lee ${ }^{1}$ and Ji Man Hong ${ }^{1 *}$ (D)

\begin{abstract}
Background: In acute large anterior circulation infarct patients with large core volume, we evaluated the role of optic nerve sheath diameter (ONSD) change rates in prediction of malignant progression.

Methods: We performed a retrospective observational study including patients with anterior circulation acute ischemic stroke with large ischemic cores from January 2010 to October 2017. Primary outcome was defined as undergoing decompressive surgery or death due to severe cerebral edema, and termed malignant progression. Patients were divided into malignant progressors and nonprogressors. Malignant progression was divided into early progression that occurred before D1 CT, and late progression that occurred afterwards. Retrospective analysis of changes in mean ONSD/eyeball transverse diameter (ETD) ratio, and midline shifting (MLS) were evaluated on serial computed tomography (CT). Through analysis of $C T$ at baseline, postprocedure, and at D1, the predictive ability of time based change in ONSD/ETD ratio in predicting malignant progression was evaluated.

Results: A total of 58 patients were included. Nineteen (32.8\%) were classified as malignant; 12 early, and 7 late progressions. In analysis of $C T_{\text {postprocedure, }}$ A $1 \mathrm{~mm} / \mathrm{hr}$. rate of change in $\mathrm{MLS}$ during the $\mathrm{CT}_{\text {baseline }}-\mathrm{CT}_{\text {postprocedure }}$ time phase lead to a 6.7 fold increased odds of early malignant progression $(p<0.05)$. For ONSD/ETD, 1\%/hr. change lead to a 1.6 fold increased odds, but this association was trending $(p=0.249)$. In the $C T_{D 1}, 1 \%$ /day change of ONSD/ETD in the $C_{\text {baseline }}-C_{D 1}$ time phase lead to a 1.4 fold increased odds of late malignant progression ( $p=$ 0.021 ) while $1 \mathrm{~mm} /$ day rate of change in MLS lead to a 1.5 fold increased odds ( $p=0.014)$.

Conclusions: The rate of ONSD/ETD changes compared to baseline at D1 CT can be a predictor of late malignant progression along with MLS. ONSD/ETD change rates evaluated at postprocedure did not predict early malignant progression.
\end{abstract}

Keywords: Cerebral infarction, Brain edema, Optic nerve, Ultrasonography, Intracranial pressure

\section{Background}

Early decompressive hemicraniectomy (DHC) performed in malignant middle cerebral artery (MCA) infarction patients can reduce mortality and improve functional

\footnotetext{
* Correspondence: dacda@hanmail.net

'Department of Neurology, Ajou University School of Medicine, 164, World cup-ro, Yeongtong-gu, Suwon-si, Gyeonggi-do 16499, Republic of Korea Full list of author information is available at the end of the article
}

outcomes [1]. In clinical practice, however, early DHC is not easy to apply to acute anterior circulation patients with large infarct core volumes. First, in DHC clinical trials, patients were randomized at least $12 \mathrm{~h}$ after onset [2], and usually did not include the patients in which intravenous (IV) thrombolysis was performed [3]. Thus patients that can be candidates for endovascular treatment may have not been included. Second, while DHC's

C C The Author(s). 2020 Open Access This article is licensed under a Creative Commons Attribution 4.0 International License, which permits use, sharing, adaptation, distribution and reproduction in any medium or format, as long as you give appropriate credit to the original author(s) and the source, provide a link to the Creative Commons licence, and indicate if changes were made. The images or other third party material in this article are included in the article's Creative Commons licence, unless indicated otherwise in a credit line to the material. If material is not included in the article's Creative Commons licence and your intended use is not permitted by statutory regulation or exceeds the permitted use, you will need to obtain permission directly from the copyright holder. To view a copy of this licence, visit http://creativecommons.org/licenses/by/4.0/. The Creative Commons Public Domain Dedication waiver (http://creativecommons.org/publicdomain/zero/1.0/) applies to the data made available in this article, unless otherwise stated in a credit line to the data. 
treatment effect in the elderly is low [4], elderly stroke patients tend to increase. Third, DHC is not without morbidity and mortality, and some argue that it results in increasing the number of surviving patients with significant functional deficits [5].

Thus imaging and clinical predictors of malignant cerebral edema have been studied to aid prediction of clinical courses. However, most of the indicators are based on initial imaging findings, such as infarct volume. On the contrary, dynamic parameters that indicate imminent herniation has not been well verified. Continuous intracranial pressure (ICP) monitoring has been indicated for such situations in severe traumatic brain injury, and an elevated ICP calls for therapeutic actions in these patients [6]. However, for malignant MCA infarction patients, invasive ICP monitoring showed inconsistent results in limited clinical reports $[7,8]$. The value of recent non-invasive ICP monitoring need yet to be validated in these patients.

Optic nerve sheath diameter (ONSD) is a noninvasive method of ICP measurement that is gathering clinical interest [9]. The communication between optic nerve subarachnoid space and the chiasmatic cistern [10] results in cerebrospinal fluid (CSF) flow toward the optic nerve subarachnoid space in situations of increased ICP. Subsequent expansion of the dural sheath causes increase in the ONSD. The dural sheath $3 \mathrm{~mm}$ behind the globe is the preferred location for measurement, as changes are most obvious at this point $[11,12]$. Dynamic changes in ONSD are thought to represent increases in ICP rather than its static values [13, 14], and thus serial bedside measurements can be practical.

Thus in the current study, in acute ischemic patients with large cores, we aimed to study the ability of computed tomography (CT)-based ONSD measurements and its time based changes in predicting malignant progression. We also aimed to compare the ONSD with midline shift (MLS), which is considered the gold standard measure for prediction of malignant edema [15] and known to be closely related to functional outcomes [16] and early mortality [17].

\section{Methods}

\section{Patient enrolment}

This study was performed using retrospective single center data from January, 2010 to October, 2017. From patients with intra/extracranial large artery occlusion evaluated for reperfusion treatments during this period, patients satisfying the below criteria were retrospectively included. (1) Anterior circulation large vessel occlusion with diffusion weighted image (DWI) infarct volume > $82 \mathrm{~mL}$ [18], (2) presentation within $6 \mathrm{~h}$ of onset, and (3) National Institutes of Health Stroke Scale (NIHSS) of same or over 15 [19]. NordicICE semi-automated software (NordicNeuroLab, Bergen, Norway) was used for volume measurements. Significant contralateral infarction was an exclusion criteria.

\section{Acute ischemic stroke management}

Intravenous or intra-arterial reperfusion treatment was performed by physician's decision. All patients at least underwent cerebral angiography. Medical methods to counter malignant cerebral edema was individualized based on a stepwise protocol [20]. Osmotherapy was performed as appropriate. Therapeutic hypothermia was performed according to previously described protocol [21].

\section{Classification of CT timepoints}

In all patients, serial CT imaging was performed to anticipate for malignant cerebral edema. The CT scans (SOMATOM Sensation 16; SOMATOM Definition Edge [128-channel] Siemens, Erlangen, Germany) were acquired with contiguous 5 -mm thick axial sections (120 $\mathrm{kV}, 270 \mathrm{mAs})$. To analyse CT based factors of predicting impending herniation, CT taken at three specific time points were analysed. Baseline CT angiography upon presentation to the emergency department was classified as $\mathrm{CT}_{\text {baseline. }} \mathrm{CT}$ taken postprocedure after endovascular treatment or angiography was classified as $\mathrm{CT}_{\text {postproce- }}$ dure. CT angiography performed on the next day of admission was classified as $\mathrm{CT}_{\mathrm{D} 1 \text {. }}$ (Fig. 1).

\section{Malignant progression and decompressive hemicraniectomy}

For all patients, DHC was usually performed based on clinical signs while managed with neurocritical care. During this period, patients that showed clinical signs such as altered mental status, flexor or extensor motor posturing, pupillary abnormality, repiratory pattern changes, eye movement impairments, or respiratory pattern abnormalities [22], with evidence of imminent herniation in CT was classified "malignant progressors [23]." Malignant progression was further divided into early malignant progression if it occurred after $\mathrm{CT}_{\text {postpro- }}$ cedure and before $\mathrm{CT}_{\mathrm{D} 1}$, and late malignant progression if it occurred after $\mathrm{CT}_{\mathrm{D} 1}$. $\mathrm{DHC}$ was performed upon consent regardless of age or whether thrombolysis was performed. Bilateral fixed pupils was a contraindication for DHC [1]. ONSD measurements were not clinically utilized.

\section{Measurement of $\mathrm{CT}$ predictors of impending herniation}

The selected CT scans were evaluated using commercial image-viewing software (Picture Archiving and Communication System; Maroview 5.3 Infinitt Co., Seoul, Republic of Korea) without access to clinical information. The ONSD and eyeball transverse diameter (ETD) were measured using "chest/abdomen" window (window 


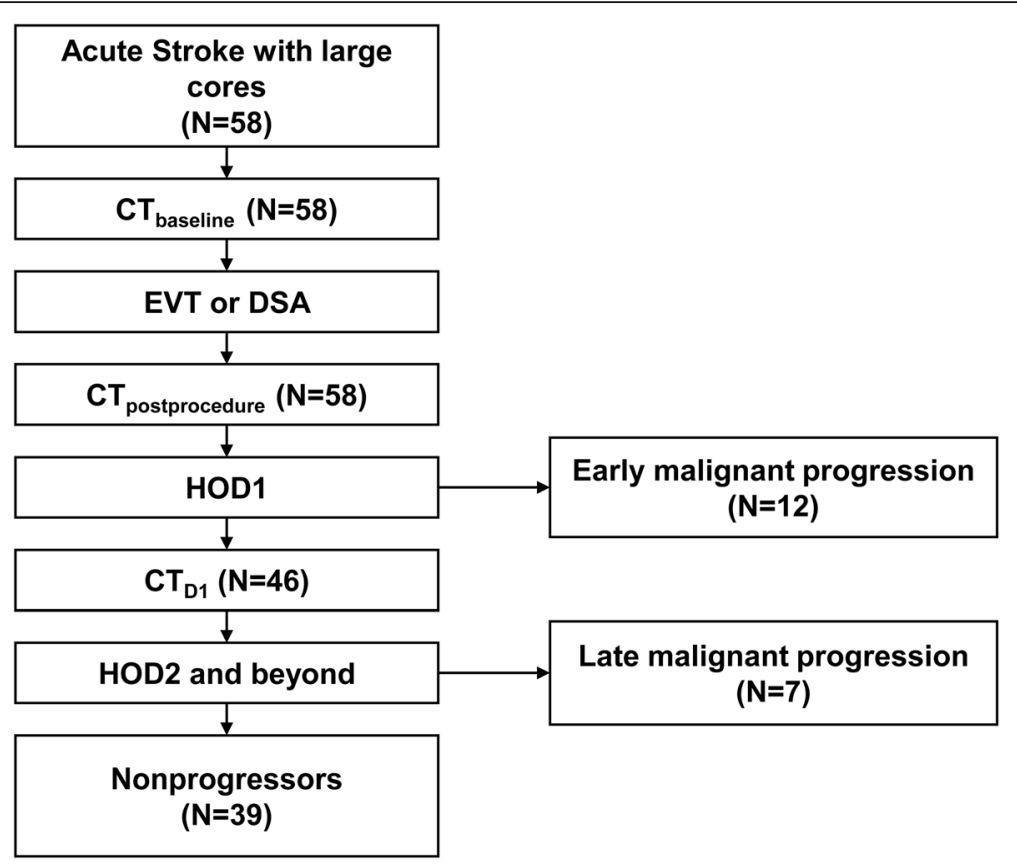

Fig. $1 \mathrm{~A}$ flow chart of the included patients. Changes in $C T$ parameters between $C T_{\text {postprocedure and }} \mathrm{C}_{\text {baseline }}$ was used to predict early malignant

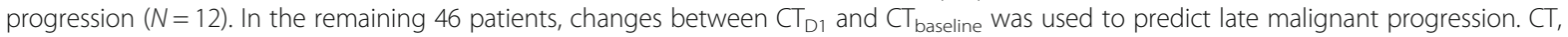
computed tomography; EVT, endovascular treatment; DSA, digital subtraction angiography; HOD, hospital day

width 300 and window level 10) [24] with fivefold magnification. The ONSD was measured $3 \mathrm{~mm}$ behind the eyeball, perpendicular to the linear axis of the optic nerve [25]. The ETD was evaluated measuring the maximal transverse diameter of the eyeball from retina to retina (Fig. 2). ONSD/ETD was calculated to correct for the ETD as a confounding factor [26]. All ONSD measurement was performed bilaterally and averaged.

In the same CT scans, MLS was measured as the degree of displacement of the septum pellucidum [27] relative to the midline in the $\mathrm{CT}$ scans. To minimize bias, MLS measurements and ONSD measurements were performed at least 2 weeks apart. Post-procedural hemorrhagic complications were classified in accordance with the European Cooperative Acute Stroke Study criteria [28].

\section{Statistical analysis}

The patients were divided into two groups based on malignant progression. Differences in clinical characteristics

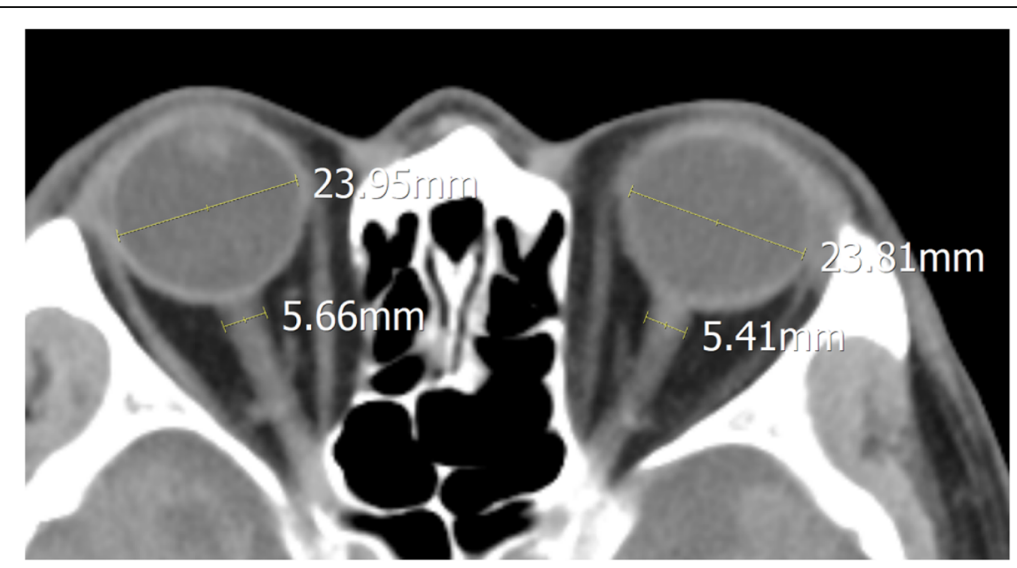

Fig. 2 Computed tomography based measurement of ONSD and ETD. The ONSD was measured at a distance of $3 \mathrm{~mm}$ behind the eyeball, immediately below the sclera in a perpendicular vector with reference to the linear axis of the nerve. The ETD was defined as the maximal transverse diameter of the eyeball measured from one side of the retina to the other. ONSD, optic nerve sheath diameter; ETD, eyeball transverse diameter 
and CT profiles were compared between the two groups. Then we performed longitudinal analysis of MLS and ONSD/ETD with a linear mixed-effects model to estimate mean levels of the parameters over time within groups from baseline to D1 of follow-up, using available data. The fixed effects were CT time points, group and group-bytime interaction effect and individual was included as a random effect. Based on this preliminary analysis, imaging analysis was performed. For imaging analysis of the

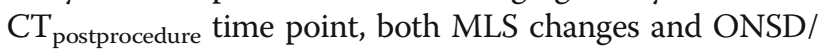
ETD changes were adjusted for time by hours from $\mathrm{CT}_{\mathrm{ba}}$ seline to $\mathrm{CT}_{\text {postprocedure }}$ to account for time based changes. Multivariate analysis was further performed to confirm the clinical significance of time corrected changes in ONSD/ETD and MLS in prediction of early malignant progression. In the $\mathrm{CT}_{\mathrm{D} 1}$ time point, both MLS changes and ONSD/ETD changes were adjusted for time by days from $\mathrm{CT}_{\text {baseline }}$ to $\mathrm{CT}_{\mathrm{D} 1}$ to account for time based changes. Multivariate analysis was further performed to confirm the clinical significance of time corrected changes in ONSD/ETD and MLS in prediction of late malignant progression. The data are presented as the mean \pm standard deviation or as the median [interquartile range]. A $P$ value less than 0.05 was considered statistically significant. Statistical analyses were performed using IBM SPSS Statistics software version 25 (IBM Corp., Armonk, NY, USA) and R software, version 3.6.2. (R Foundation for Statistical Computing, Vienna, Austria).

\section{Results}

\section{Clinical characteristics and initial imaging profiles}

( $\left.\mathrm{CT}_{\text {baseline }}\right)$

In the overall 58 patients, early malignant progression occurred between $\mathrm{CT}_{\text {postprocedure }}$ and $\mathrm{CT}_{\mathrm{D} 1}$ (within 12 to $24 \mathrm{~h}$ ) in 12 patients. In 7 patients, late malignant progression occurred after $\mathrm{CT}_{\mathrm{D} 1}$. Overall, malignant progression occurred in 19 and 39 could be managed with medical measures to reduce cerebral edema (Fig. 1). Clinical characteristics and outcomes of 19 patients classified as malignant progression are described in Table 1.

In comparison of clinical characteristics, CT angiography based occlusion location was significantly different between two groups $(p=0.015)$, with complex $\mathrm{T}$ type occlusions [29] much more common in the malignant progressors compared to nonprogressors (52.6\% vs. $15.4 \%)$. Initial DWI volume was also significantly higher in the malignant progressors (237 \pm $109 \mathrm{ml}$ vs. $144 \pm 43 \mathrm{ml}, p=0.002$ ). Endovascular treatment was performed in all malignant progressors, while it was performed in $74.4 \%$ of nonprogressors $(p=0.015)$. The functional outcomes were significantly different $(p=0.014)$, with $84.2 \%$ resulting in modified Rankin Scale of 5-6 in the malignant progressors, while the percentage was $41.0 \%$ in the nonprogressors. There were no differences regarding ONSD, ONSD/ETD ratio, or MLS when the $\mathrm{CT}_{\text {baseline }}$ was compared (Table 2).

Table 1 Clinical characteristics of 19 patients with malignant progression and their classification process

\begin{tabular}{|c|c|c|c|c|c|}
\hline No. & DWI volume $(\mathrm{ml})$ & $\mathrm{CT}_{\text {baseline }}$ to malignant progression $(\mathrm{hr})$ & Classification process & Decompression & $3 \mathrm{~m} \mathrm{mRS}$ \\
\hline 1 & 124 & 9.4 & Pupillary changes & $\mathrm{N}$ & 6 \\
\hline 2 & 125 & 17.8 & Pupillary changes & Y & 6 \\
\hline 3 & 129 & 21.2 & Imaging based decision & Y & 3 \\
\hline 4 & 96 & 17.4 & Pupillary changes & $\mathrm{N}$ & 6 \\
\hline 5 & 116 & 8.5 & Pupillary changes & $\mathrm{N}$ & 6 \\
\hline 6 & 148 & 149.8 & Pupillary changes & $\mathrm{N}$ & 6 \\
\hline 7 & 179 & 96.0 & Pupillary changes & Y & 5 \\
\hline 8 & 218 & 28.9 & Pupillary changes & Y & 5 \\
\hline 9 & 232 & 46.4 & Pupillary changes & Y & 4 \\
\hline 10 & 244 & 13.3 & Pupillary changes & N & 6 \\
\hline 11 & 304 & 19.7 & Pupillary changes & Y & 5 \\
\hline 12 & 308 & 5.6 & Imaging based decision & N & 6 \\
\hline 13 & 319 & 4.0 & Coma & Y & 6 \\
\hline 14 & 364 & 13.2 & Pupillary changes & $\mathrm{N}$ & 6 \\
\hline 15 & 391 & 7.8 & Pupillary changes & Y & 6 \\
\hline 16 & 475 & 5.4 & Imaging based decision & Y & 6 \\
\hline 17 & 343 & 5.4 & Imaging based decision & Y & 4 \\
\hline 18 & 155 & 21.0 & Pupillary changes & $\mathrm{N}$ & 6 \\
\hline 19 & 227 & 7.0 & Pupillary changes & $\mathrm{N}$ & 6 \\
\hline
\end{tabular}

DWI Diffusion weighted imaging, CT Computed tomography, mRS Modified Rankin Scale 
Longitudinal analysis of MLS and ONSD/ETD with a linear mixed-effects model

When the trajectories of MLS and ONSD/ETD was compared, MLS increased at a steeper angle in the malignant progressors compared to the nonprogressors throughout the timepoints. For ONTD/ETD, it increased at a steeper angle in the malignant progressors up to postprocedure time point, and then this difference was maintained up to the D1 time point. However, a transient rise was seen in the nonprogressors at the postprocedure time point, decreasing the gap at this point (Fig. 3). In the longitudinal analysis with a linear fixed affects model, the time and group-by-time interaction effects were significant $(P<0.05)$, showing that the changes in MLS and ONSD/ETD ratio according to time points were significantly different in the nonprogressors and malignant progressors.

\section{Comparison of $\mathrm{CT}_{\text {postprocedure in prediction of early }}$ malignant progression}

Early malignant progression occurred in 12 patients with a mean time from $\mathrm{CT}_{\text {baseline }}$ to malignant progression of $10.8 \pm 7.4 \mathrm{~h}$. In the $\mathrm{CT}_{\text {postprocedure, the patients that }}$ showed early malignant progression showed significant

Table 2 Differences in clinical characteristics and baseline imaging profiles according to dichotomized clinical course based on malignant progression

\begin{tabular}{|c|c|c|c|}
\hline & $\begin{array}{l}\text { Malignant progressors } \\
(n=19)\end{array}$ & $\begin{array}{l}\text { Nonprogressors } \\
(n=39)\end{array}$ & $P$-value \\
\hline \multicolumn{4}{|l|}{ Clinical characteristics } \\
\hline Sex, male & $9(47.4 \%)$ & $22(56.4 \%)$ & 0.517 \\
\hline Age & $65 \pm 19$ & $69 \pm 13$ & 0.407 \\
\hline Hypertension & $15(75.0 \%)$ & $24(60.0 \%)$ & 0.251 \\
\hline Diabetes mellitus & $8(40.0 \%)$ & $10(25.0 \%)$ & 0.232 \\
\hline Onset to door (min) & $195 \pm 240$ & $173 \pm 182$ & 0.511 \\
\hline Hypothermia & $10(52.6 \%)$ & $21(53.8 \%)$ & 0.931 \\
\hline Thrombolysis & $12(63.2 \%)$ & $29(74.4 \%)$ & 0.379 \\
\hline Endovascular treatment & $19(100.0 \%)$ & $29(74.4 \%)$ & 0.015 \\
\hline Successful reperfusion & $11(57.9 \%)$ & $22(56.4 \%)$ & 0.915 \\
\hline Outcomes (3 month mRS) & & & 0.014 \\
\hline 0 & $0(0.0 \%)$ & $2(5.1 \%)$ & \\
\hline 1 & $0(0.0 \%)$ & $2(5.1 \%)$ & \\
\hline 2 & $0(0.0 \%)$ & $5(12.8 \%)$ & \\
\hline 3 & $1(5.3 \%)$ & $4(10.3 \%)$ & \\
\hline 4 & $2(10.5 \%)$ & $10(25.6 \%)$ & \\
\hline 5 & $3(15.8 \%)$ & $9(23.1)$ & \\
\hline 6 & $13(68.4 \%)$ & 7 (17.9\%) & \\
\hline \multicolumn{4}{|l|}{$\mathrm{CT}_{\text {baseline }}$} \\
\hline CT angiography occlusion & & & 0.015 \\
\hline Complex T & $10(52.6 \%)$ & $6(15.4 \%)$ & \\
\hline Simple T & $4(21.1 \%)$ & $11(28.2 \%)$ & \\
\hline MCA M1 & $3(15.8 \%)$ & $14(35.9 \%)$ & \\
\hline Tandem & $0(0.0 \%)$ & 7 (17.9\%) & \\
\hline Proximal ICA & $1(5.3 \%)$ & $0(0.0 \%)$ & \\
\hline Dissection & $1(5.3 \%)$ & $1(2.6 \%)$ & \\
\hline Initial DWI volume (ml) & $237 \pm 109$ & $144 \pm 43$ & 0.002 \\
\hline ONSD (mm) & $5.17 \pm 0.55$ & $5.37 \pm 0.63$ & 0.233 \\
\hline ONSD/ETD ratio & $22.84 \pm 2.33 \%$ & $23.60 \pm 2.83 \%$ & 0.316 \\
\hline MLS (mm) & $0.44 \pm 0.78$ & $0.37 \pm 0.95$ & 0.789 \\
\hline
\end{tabular}

Values are presented as mean \pm SD, number (\%), or median (interquartile range) mRS Modified Rankin Scale, CT Computed tomography, MCA Middle cerebral artery, ICA Internal carotid artery, DWI Diffusion weighted images, ONSD Optic nerve sheath diameter, ETD Eyeball transverse diameter, MLS Midline shift 

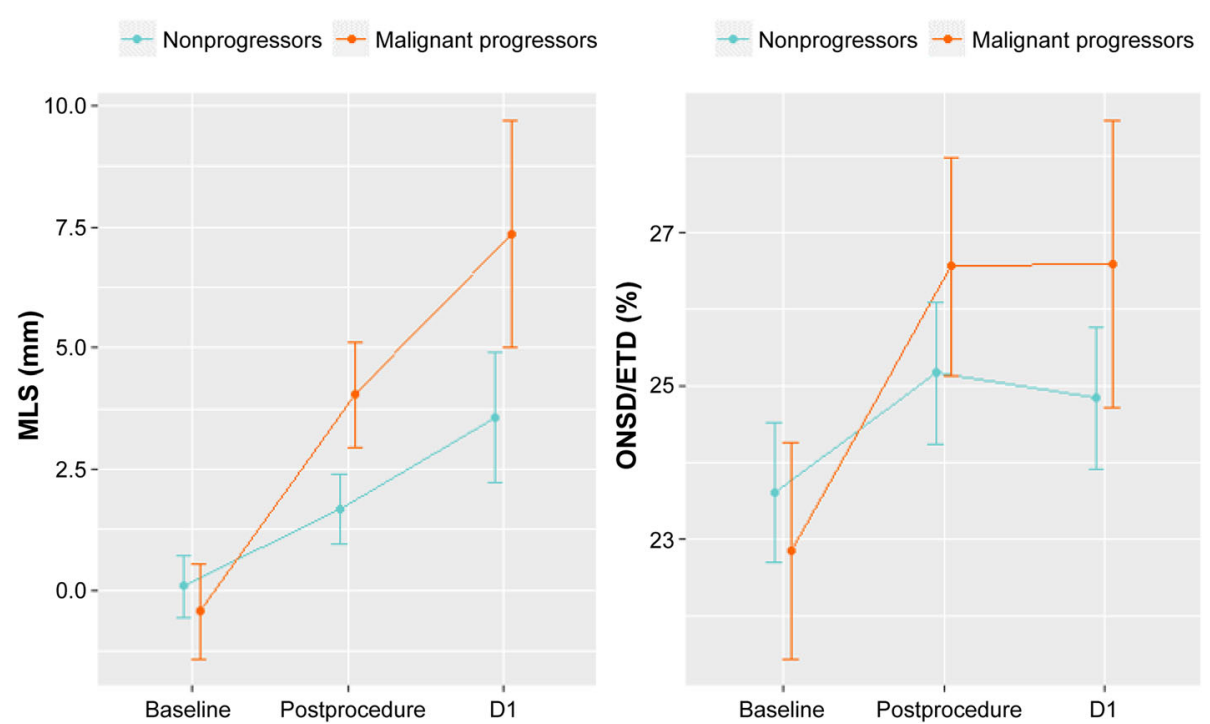

Fig. 3 Trajectories of MLS (left panel) and ONSD/ETD (right panel) of follow-up. The time series change in MLS and ONSD/ETD, adjusted for age, sex and DWI volume, are plotted from baseline to D1. The rate of increase of MLS is constantly higher in the malignant progressors throughout the time periods. For ONSD/ETD ratio, it is increased at the postprocedure time point, and this difference is maintained. Due to a slight increase of ONSD/ETD ratio in the postprocedure time point in the nonprogressors, this difference becomes more evident at the D1 timepoint. Error bars represent 95\% Cls. MLS, midline shift; ONSD, optic nerve sheath diameter; ETD, eyeball transverse diameter

increases in MLS $(6.08 \pm 4.82 \mathrm{~mm}$ vs. $1.69 \pm 2.21 \mathrm{~mm}$, $p=0.009)$, and more frequent hemorrhagic transformation $(p<0.001)$. Changes in ONSD/ETD ratio was not significantly different $(3.70 \pm 2.78 \%$ vs. $1.89 \pm 3.37 \%, p=$ 0.093) (Table 3). When the changes in MLS and ONSD/ ETD were normalized by time from $\mathrm{CT}_{\text {baseline }}$ to $\mathrm{CT}_{\text {post- }}$ procedure, the rate of change in MLS was associated with increased odds of early malignant progression with age, sex, and initial infarct volume as covariables. A $1 \mathrm{~mm} / \mathrm{Hr}$ rate of change in MLS during this time phase lead to a 6.67 fold increase in the odds of early malignant progression $(p<0.05)$. For ONSD/ETD, $1 \% / \mathrm{Hr}$ change in this time phase lead to a 1.64 fold increase in the odds of early malignant progression, but this association was trending $(p>0.05)$ (Table 4$)$.

\section{Comparison of $\mathrm{CT}_{\mathrm{D} 1}$ in prediction of late malignant progression}

Late malignant progression occurred in 7 patients with a mean time from $\mathrm{CT}_{\text {baseline }}$ to malignant progression of $52.6 \pm 51.5 \mathrm{~h}$. In the $\mathrm{CT}_{\mathrm{D} 1}$, the patients that showed early malignant progression showed significant Changes in ONSD/ETD ratio compared to baseline $(3.60 \pm 2.67 \%$ vs. $1.05 \pm 2.69 \%, p=0.025)$. However, MLS $(p=0.054)$ or hemorrhagic patterns $(p=0.838)$ were not different between the groups at this timepoint (Table 3). When the changes in MLS and ONSD/ETD were normalized by time from $\mathrm{CT}_{\text {baseline }}$ to $\mathrm{CT}_{\mathrm{D} 1}$, the rate of change from baseline to CT ${ }_{\text {D1 }}$ in both ONSD/ETD and MLS was associated with increased odds of early malignant progression with age, sex, and initial infarct volume as co-variables. For ONSD/ETD, 1\%/Day change in this time phase lead to a 1.38 fold increase in the odds of late malignant progression $(p=0.021)$. For MLS, $1 \mathrm{~mm} /$ Day rate of change in MLS during this time phase lead to a 1.47 fold increase in the odds of late malignant progression $(p=0.014)$. (Table 4$)$.

\section{Discussion}

The results of this study shows that in acute stroke patients with malignant MCA profile, early increases in ICP evaluated by ONSD/ETD increase rates at D1 can predict future malignant progression. When evaluated postprocedure, its rate of increase was not predictive of early malignant progression. Rate of changes in MLS could predict impending malignant progression both at the postprocedure and D1 time points. These dynamic markers seem to be especially valuable when evaluated in the early phase of treatment, and interpreted by evolution time.

Through utilizing time based changes of ONSD/ETD in a patient group that was primarily managed with neurocritical care with late decisions for $\mathrm{DHC}$, the main result of this study shows that elevations in ONSD/ETD ratio compared to baseline within 1 day can predict malignant progression, and may call for therapeutic interventions. This is consistent with recent reports that emphasize the value of dynamic changes in ONSD [30]. Such clinical application is identical to invasive ICP monitoring in traumatic brain injury, in which elevations 
Table 3 Comparison of optic nerve sheath diameter related parameters, midline shift, and hemorrhagic patterns on postprocedure and D1 CT images according to early and late malignant progression

\begin{tabular}{|c|c|c|c|}
\hline \multicolumn{4}{|l|}{$\mathrm{CT}_{\text {postprocedure }}$} \\
\hline & $\begin{array}{l}\text { Early malignant progressors } \\
(n=12)\end{array}$ & $\begin{array}{l}\text { Nonprogressors } \\
(n=46)\end{array}$ & $P$-value \\
\hline $\mathrm{CT}_{\text {baseline }}$ to malignant progression, $\mathrm{hr}$ & $10.8 \pm 7.4$ & & \\
\hline$\Delta$ time $\left(C T_{\text {postprocedure }}-\mathrm{CT}_{\text {baseline }}\right), \mathrm{hr}$ & $3.9 \pm 1.2$ & $4.2 \pm 3.2$ & 0.716 \\
\hline ONSD (mm) & $5.92 \pm 0.56$ & $5.81 \pm 0.71$ & 0.599 \\
\hline ONSD/ETD ratio, \% & $26.24 \pm 1.99$ & $25.45 \pm 2.9$ & 0.382 \\
\hline$\Delta$ ONSD/ETD ratio, $\%$ & $3.70 \pm 2.78$ & $1.89 \pm 3.37$ & 0.093 \\
\hline MLS (mm) & $6.08 \pm 4.82$ & $1.69 \pm 2.21$ & 0.009 \\
\hline Hemorrhagic pattern & & & $<0.001$ \\
\hline No hemorrhage & $0(0.0 \%)$ & $14(30.4 \%)$ & \\
\hline HI 1 & $0(0.0 \%)$ & $10(21.7 \%)$ & \\
\hline $\mathrm{HI} 2$ & $1(8.3 \%)$ & $12(26.1 \%)$ & \\
\hline PH 1 & $4(33.3 \%)$ & $4(8.7 \%)$ & \\
\hline $\mathrm{PH} 2$ & $7(58.3 \%)$ & $6(13.0 \%)$ & \\
\hline \multicolumn{4}{|l|}{$\mathrm{CT}_{\mathrm{D} 1}$} \\
\hline & $\begin{array}{l}\text { Late malignant progressors } \\
(n=7)\end{array}$ & $\begin{array}{l}\text { Nonprogressors } \\
(n=39)\end{array}$ & $P$-value \\
\hline $\mathrm{CT}_{\text {baseline }}$ to malignant progression, $\mathrm{hr}$ & $52.6 \pm 51.5$ & & \\
\hline$\Delta$ time $\left(\mathrm{CT}_{\mathrm{D} 1}-\mathrm{CT}_{\text {baseline }}\right), \mathrm{hr}$ & $20.0 \pm 11.3$ & $27.4 \pm 11.9$ & 0.136 \\
\hline ONSD (mm) & $6.10 \pm 0.36$ & $5.67 \pm 0.74$ & 0.138 \\
\hline ONSD/ETD ratio, \% & $26.93 \pm 2.64$ & $24.83 \pm 2.94$ & 0.085 \\
\hline$\Delta$ ONSD/ETD ratio, $\%$ & $3.60 \pm 2.67 \%$ & $1.05 \pm 2.69$ & 0.025 \\
\hline MLS (mm) & $6.55 \pm 2.87$ & $3.64 \pm 3.68$ & 0.054 \\
\hline Hemorrhagic pattern & & & 0.819 \\
\hline No hemorrhage & $3(42.9 \%)$ & $22(56.4 \%)$ & \\
\hline HI 1 & $1(14.3 \%)$ & 7 (17.9\%) & \\
\hline $\mathrm{HI} 2$ & 1 (14.3\%) & $4(10.3 \%)$ & \\
\hline PH 1 & $2(28.6 \%)$ & $6(15.4 \%)$ & \\
\hline $\mathrm{PH} 2$ & $0(0.0 \%)$ & $0(0.0 \%)$ & \\
\hline
\end{tabular}

Values are presented as mean $\pm S D$, number $(\%)$, or median (interquartile range)

CT Computed tomography angiography, ONSD Optic nerve sheath diameter, ETD Eyeball transverse diameter, MLS Midline shift, HI Hemorrhagic infarct, $P H$ Parenchymal hematoma

in ICP above $20 \mathrm{mmHg}$ can predict clinical deterioration and calls for therapeutic intervention [31]. Further accumulating evidence of the value of dynamic changes in ONSD will support active use of ONSD measurements in neurocritical care.

The rate of ONSD/ETD fail to prove its significance in predicting malignant progression at the postprocedure time point. ICP is subject to changes according to changes in posture [32], or pain [33] related to acute procedures. The postprocedure period is exposed to such factors, and may have hampered the significane of ONSD/ETD at this period. This is supported by the fact that the mean trajectory of ONSD/ETD increases at the postprocedural time point and then decreases at D1 in the nonprogressors. Thus the optimal time to evaluate ICP changes compared to baseline may be when the patient is stabilized, likely at an intensive care unit setting.

Theoretically, prolonged elevations in ICP suggest failure of compensatory mechanism, resulting in decreased cerebral perfusion pressure and cerebral blood flow, increase in ischemia, and fulminant progression of herniation [34]. However, previous studies utilizing intraparenchymal ICP sensors [8] or epidural probes [7] in malignant cerebral edema suggested that pupillary abnormalities and severe brainstem compression may be present despite normal ICP values. This may possibly be due to the distance between the site of ICP probe insertion and the site of herniation, because the pressure 
Table 4 Logistic regression models for prediction of early and late malignant progression with changes in MLS and ONSD/ETD ratio normalized by time interval

\begin{tabular}{|c|c|c|c|c|c|}
\hline Variables & OR $(95 \% \mathrm{Cl})$ & $P$-value & Variables & OR $(95 \% \mathrm{Cl})$ & $P$-value \\
\hline \multicolumn{6}{|l|}{ Early malignant progression } \\
\hline Change from baseline in MLS, $\mathrm{mm} / \mathrm{hr}{ }^{a}$ & $6.67(1.51-29.53)$ & 0.012 & Change from baseline in ONSD/ETD, \%/hr. ${ }^{a}$ & $1.64(0.71-3.79)$ & 0.249 \\
\hline Age & $1.08(0.98-1.18)$ & 0.114 & Age & $1.03(0.97-1.09)$ & 0.379 \\
\hline Sex, male & $0.77(0.09-6.53)$ & 0.814 & Sex, male & $0.77(0.13-4.77)$ & 0.783 \\
\hline Initial DWI volume & $1.02(1.01-1.04)$ & 0.006 & Initial DWI volume & $1.02(1.01-1.03)$ & 0.001 \\
\hline \multicolumn{6}{|l|}{ Late malignant progression } \\
\hline Change from baseline in $M L S, m m /$ day ${ }^{b}$ & $1.47(1.08-2.01)$ & 0.014 & Change from baseline in ONSD/ETD, \%/day ${ }^{b}$ & $1.38(1.05-1.81)$ & 0.021 \\
\hline Age & $0.93(0.85-1.03)$ & 0.178 & Age & $0.97(0.9-1.04)$ & 0.345 \\
\hline Sex, male & $0.24(0.02-3.74)$ & 0.308 & Sex, male & $0.78(0.11-5.82)$ & 0.811 \\
\hline Initial DWI volume & $1.02(0.99-1.04)$ & 0.136 & Initial DWI volume & $1.02(1.00-1.04)$ & 0.082 \\
\hline
\end{tabular}

${ }^{a}$ Rate of change between $\mathrm{CT}_{\text {baseline }}$ and $\mathrm{CT}_{\text {postprocedure }}$

${ }^{\mathrm{b}}$ Rate of change between $\mathrm{CT}_{\text {baseline }}$ and $\mathrm{CT}_{\mathrm{D} 1}$

MLS Midline shift, ONSD Optic nerve sheath diameter, ETD Eyeball transverse diameter, OR Odds ratio, DWI Diffusion weighted imaging

gradient force is inversely proportional to the distance [20]. In this regard, ONSD changes may be a superior way of ICP monitoring for malignant stroke patients, for it physiologically directly represents intraventricular pressure [10], which is gold standard. Future studies with invasive ICP monitoring are needed to answer this question.

MLS is a well validated marker of malignant edema [15], and it is closely related with functional outcomes [16] and early mortality [17]. Some institutions have used a midline shift of more than $5 \mathrm{~mm}$ as an indicator of surgical management [8]. The rate of MLS increases according to time could predict both early and late malignant progression. However, when interpreted as an absolute value, there were no significant difference between the two groups in the D1 CT. This result shows that its evolution speed is more important than MLS as an absolute value. For example, MLS of $5 \mathrm{~mm}$ observed $4 \mathrm{~h}$ after admission and $5 \mathrm{~mm}$ observed 5 days after admission differs in clinical significance, and it is highly likely that the former case will need surgical intervention. To represent such temporal changes in time, variables such as infarct growth rate [35] is also being increasingly used.

The clinical role of ONSD based noninvasive measurements of ICP in ischemic stroke is increasingly gathering attention. In previous literatures, it has been shown that ONSD increases in early ischemic stroke compared to normal controls [36]. In ischemic stroke subtypes, a total anterior circulation infarct type was associated with elevations in ONSD compared to control and other stroke subtypes [36]. It has been further shown that ONSD can differentiate ischemic stroke and hemoarrhagic stroke with moderate predictive power [37]. A recent study compared the ONSD/ETD in initial CT scans of malignant MCA patients and age and gender matched controls. The authors could show that malignant MCA patients showed significantly higher ONSD/ETD ratio [38]. Such results and the current study results may advocate for CT or ultrasound based measurements of ONSD for ischemic stroke as an easily accessible measure to guide therapy.

There are some limitations to the study. First is that invasive ICP monitoring was not performed. This is due to current practice guidelines that do not recommend invasive monitoring in ischemic stroke [20]. Instead, the current results suggest that ONSD/ETD ratio measurements may be used as an alternative method for ICP measurements in these patients. However, while the association between invasive ICP values and ONSD/ETD ratio have been proven in previous studies, its correlation in ischemic stroke patients still need to be proven. Second, in evidence based practice, there are some factors that limit generalization of this retrospective study. Because a large number of patients were included before 2015, patient selection criteria for endovascular treatment was permissive. Furthermore, osmotherapy, sedation, and hypothermia are still of unproven benefit. However, malignant stroke is a rare clinical situation, and the authors think that this study results can guide tailored neurocritical management of such patients. Third, since the usual thickness for ONSD nears $5 \mathrm{~mm}$, the $5 \mathrm{~mm}$ slice thickness CT protocol may introduce a certain margin of error due to partial volume effect [39]. Nevertheless, previous studies have shown the usefulness of ONSD measurements in $5 \mathrm{~mm}$ CT thickness [40], as was the result in our study. Fourth, because of its timely accessibility and utility at bedside, ultrasound based ONSD measurements may be most preferable to guide therapy in ischemic stroke patients treated with intensive medical therapy to control cerebral edema. In this regard, larger prospective studies are needed to confirm 
the value of serial ONSD guided managements in malignant cerebral infarcts, possibly by ultrasound at the bedside.

\section{Conclusion}

In conclusion, in acute stroke patients with malignant infarct cores, an increase in ONSD/ETD ratio compared to baseline increases the odds of malignant progression, and may be used as a marker for emergent therapeutic interventions.

\section{Abbreviations}

CSF: Cerebrospinal fluid; CT: Computed tomography; DHC: Decompressive hemicraniectomy; DWI: Diffusion weighted image; ETD: Eyeball transverse diameter; ICP: Intracranial pressure; MCA: Middle cerebral artery; MLS: Midline shift; NIHSS: National Institutes of Health Stroke Scale; ONSD: Optic nerve sheath diameter

\section{Acknowledgements}

Not applicable.

\section{Authors' contributions}

$\mathrm{SL}$ and $\mathrm{JH}$ contributed to the conception and design of the work. SL, JH, and $J \mathrm{~L}$ contributed to the acquisition, analysis and interpretation of data. JP and $\mathrm{BP}$ contributed to analysis and interpretation of data. SL, JH, MC, and SL contributed to the draft of the work and substantial intellectual revisions. The author(s) read and approved the final manuscript.

\section{Funding}

Not applicable.

\section{Availability of data and materials}

The datasets used and/or analysed during the current study are available upon reasonable request to the corresponding author.

\section{Ethics approval and consent to participate}

The data collection protocol was approved by the Ajou University Hospital Institutional Review Board (MED-MDB-20-372) and implemented in accordance with the ethical standards of the 1964 Declaration of Helsinki and its later amendments. The need for written informed consent was waived given the retrospective nature of the study.

\section{Consent for publication}

Not applicable.

\section{Competing interests}

All authors have nothing to disclose.

\section{Author details}

'Department of Neurology, Ajou University School of Medicine, 164, World cup-ro, Yeongtong-gu, Suwon-si, Gyeonggi-do 16499, Republic of Korea. ${ }^{2}$ Office of Biostatistics, Medical Research Collaborating Center, Ajou Research Institute for Innovative Medicine, Ajou University Medical Center, Suwon, Republic of Korea. ${ }^{3}$ Department of Biomedical Informatics, Ajou University School of Medicine, Suwon, Republic of Korea.

Received: 14 January 2020 Accepted: 14 September 2020

Published online: 22 September 2020

\section{References}

1. Vahedi K, Hofmeijer J, Juettler E, Vicaut E, George B, Algra A, Amelink GJ, Schmiedeck P, Schwab S, Rothwell PM, et al. Early decompressive surgery in malignant infarction of the middle cerebral artery: a pooled analysis of three randomised controlled trials. Lancet Neurol. 2007;6(3):215-22.

2. Vahedi K, Vicaut E, Mateo J, Kurtz A, Orabi M, Guichard JP, Boutron C,

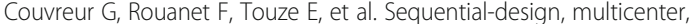
randomized, controlled trial of early decompressive craniectomy in malignant middle cerebral artery infarction (DECIMAL trial). Stroke. 2007; 38(9):2506-17.

3. Hofmeijer J, Kappelle LJ, Algra A, Amelink GJ, van Gijn J, van der Worp HB investigators $\mathrm{H}$. Surgical decompression for space-occupying cerebral infarction (the Hemicraniectomy after middle cerebral artery infarction with life-threatening edema trial [HAMLET]): a multicentre, open, randomised trial. Lancet Neurol. 2009;8(4):326-33.

4. Juttler E, Unterberg A, Woitzik J, Bosel J, Amiri H, Sakowitz OW, Gondan M, Schiller P, Limprecht R, Luntz S, et al. Hemicraniectomy in older patients with extensive middle-cerebral-artery stroke. N Engl J Med. 2014;370(12): 1091-100.

5. Puetz V, Campos CR, Eliasziw M, Hill MD, Demchuk AM, Calgary Stroke P. Assessing the benefits of hemicraniectomy: what is a favourable outcome? Lancet Neurol. 2007;6(7):580 author reply 580-581.

6. Brain Trauma F, American Association of Neurological S, Congress of Neurological S, Joint Section on N, Critical Care AC, Bratton SL, Chestnut RM, Ghajar J, McConnell Hammond FF, Harris OA, et al. Guidelines for the management of severe traumatic brain injury. VII. Intracranial pressure monitoring technology. J Neurotrauma. 2007;24(Suppl 1):S45-54

7. Schwab S, Aschoff A, Spranger M, Albert F, Hacke W. The value of intracranial pressure monitoring in acute hemispheric stroke. Neurology. 1996:47(2):393-8.

8. Poca MA, Benejam B, Sahuquillo J, Riveiro M, Frascheri L, Merino MA, Delgado P, Alvarez-Sabin J. Monitoring intracranial pressure in patients with malignant middle cerebral artery infarction: is it useful? J Neurosurg. 2010; 112(3):648-57.

9. Strumwasser A, Kwan RO, Yeung L, Miraflor E, Ereso A, Castro-Moure F, Patel A, Sadjadi J, Victorino GP. Sonographic optic nerve sheath diameter as an estimate of intracranial pressure in adult trauma. J Surg Res. 2011;170(2): 265-71

10. Killer HE, Laeng HR, Flammer J, Groscurth P. Architecture of arachnoid trabeculae, pillars, and septa in the subarachnoid space of the human optic nerve: anatomy and clinical considerations. Br J Ophthalmol. 2003;87(6):777_ 81.

11. Liu D, Kahn M. Measurement and relationship of subarachnoid pressure of the optic nerve to intracranial pressures in fresh cadavers. Am J Ophthalmol. 1993;116(5):548-56.

12. Hansen HC, Helmke K. The subarachnoid space surrounding the optic nerves. An ultrasound study of the optic nerve sheath. Surg Radiol Anat 1996;18(4):323-8.

13. Hansen $\mathrm{HC}$, Helmke K. Validation of the optic nerve sheath response to changing cerebrospinal fluid pressure: ultrasound findings during intrathecal infusion tests. J Neurosurg. 1997:87(1):34-40.

14. Gangemi M, Cennamo G, Maiuri F, D'Andrea F. Echographic measurement of the optic nerve in patients with intracranial hypertension. Neurochirurgia. 1987;30(2):53-5

15. Ropper $\mathrm{AH}$. Lateral displacement of the brain and level of consciousness in patients with an acute hemispheral mass. N Engl J Med. 1986;314(15):953-8.

16. Ross DA, Olsen WL, Ross AM, Andrews BT, Pitts LH. Brain shift, level of consciousness, and restoration of consciousness in patients with acute intracranial hematoma. J Neurosurg. 1989:71(4):498-502.

17. Kimberly WT, Sheth KN. Approach to severe hemispheric stroke. Neurology. 2011:76(7 Suppl 2):S50-6

18. Thomalla G, Hartmann F, Juettler E, Singer OC, Lehnhardt FG, Kohrmann M, Kersten JF, Krutzelmann A, Humpich MC, Sobesky J, et al. Prediction of malignant middle cerebral artery infarction by magnetic resonance imaging within 6 hours of symptom onset: a prospective multicenter observational study. Ann Neurol. 2010;68(4):435-45

19. Krieger DW, Demchuk AM, Kasner SE, Jauss M, Hantson L. Early clinical and radiological predictors of fatal brain swelling in ischemic stroke. Stroke. 1999;30(2):287-92.

20. Jeon SB, Koh Y, Choi HA, Lee K. Critical care for patients with massive ischemic stroke. J Stroke. 2014;16(3):146-60

21. Hong JM, Lee JS, Song HJ, Jeong HS, Choi HA, Lee K. Therapeutic hypothermia after recanalization in patients with acute ischemic stroke. Stroke. 2014;45(1):134-40.

22. Mayer SA, Coplin WM, Raps EC. Cerebral edema, intracranial pressure, and herniation syndromes. J Stroke Cerebrovasc Dis. 1999;8(3):183-91.

23. Lee SJ, Lee KS, Lee JS, Choi MH, Lee SE, Hong JM. Primary neurocritical care involving therapeutic hypothermia for acute ischemic stroke patients with malignant infarct cores. J Neurocrit Care. 2019;12(1):30-6. 
24. Sekhon MS, Griesdale DE, Robba C, McGlashan N, Needham E, Walland K, Shook AC, Smielewski P, Czosnyka M, Gupta AK, et al. Optic nerve sheath diameter on computed tomography is correlated with simultaneously measured intracranial pressure in patients with severe traumatic brain injury. Intensive Care Med. 2014;40(9):1267-74.

25. Legrand A, Jeanjean P, Delanghe F, Peltier J, Lecat B, Dupont H. Estimation of optic nerve sheath diameter on an initial brain computed tomography scan can contribute prognostic information in traumatic brain injury patients. Crit Care. 2013;17(2):R61.

26. Vaiman M, Gottlieb P, Bekerman I. Quantitative relations between the eyeball, the optic nerve, and the optic canal important for intracranial pressure monitoring. Head Face Med. 2014;10:32.

27. The Brain Trauma Foundation. The American Association of Neurological Surgeons. The Joint Section on Neurotrauma and Critical Care. Computed tomography scan features. J Neurotrauma. 2000;17(6-7):597-627.

28. Fiorelli M, Bastianello S, von Kummer R, del Zoppo GJ, Larrue V, Lesaffre E, Ringleb AP, Lorenzano S, Manelfe C, Bozzao L. Hemorrhagic transformation within 36 hours of a cerebral infarct: relationships with early clinical deterioration and 3-month outcome in the European cooperative acute Stroke study I (ECASS I) cohort. Stroke. 1999;30(11):2280-4.

29. Lee SU, Hong JM, Kim SY, Bang OY, Demchuk AM, Lee JS. Differentiating carotid terminus occlusions into two distinct populations based on Willisian collateral status. J Stroke. 2016;18(2):179-86.

30. Wang $L$, Chen LM, Chen Y, Bao LY, Zheng NN, Wang YZ, Xing YQ. Ultrasonography assessments of optic nerve sheath diameter as a noninvasive and dynamic method of detecting changes in intracranial pressure. JAMA Ophthalmol. 2018;136(3):250-6

31. Brain Trauma F, American Association of Neurological S, Congress of Neurological S, Joint Section on N, Critical Care AC, Bratton SL, Chestnut RM, Ghajar J, FF MCH, Harris OA, et al. Guidelines for the management of severe traumatic brain injury. VIII. Intracranial pressure thresholds. J Neurotrauma. 2007;24(Suppl 1):S55-8.

32. Andresen $M$, Hadi A, Petersen $L G$, Juhler M. Effect of postural changes on ICP in healthy and ill subjects. Acta Neurochir. 2015;157(1):109-13.

33. Bellieni CV, Burroni A, Perrone S, Cordelli DM, Nenci A, Lunghi A, Buonocore G. Intracranial pressure during procedural pain. Biol Neonate. 2003;84(3): 202-5.

34. Smith M. Monitoring intracranial pressure in traumatic brain injury. Anesth Analg. 2008:106(1):240-8.

35. Kamran S, Salam A, Akhtar N, Alboudi A, Kamran K, Singh R, Amir N, Inshasi J, Qidwai U, Malik RA, et al. Factors that can help select the timing for Decompressive Hemicraniectomy for malignant MCA Stroke. Transl Stroke Res. 2018;9(6):600-7.

36. Gokcen E, Caltekin I, Savrun A, Korkmaz H, Savrun ST, Yildirim G. Alterations in optic nerve sheath diameter according to cerebrovascular disease subgroups. Am J Emerg Med. 2017;35(11):1607-11.

37. Manouchehrifar M, Lakestani M, Kashani P, Safari S. Sonographic diameter of optic nerve sheath in differentiation of ischemic and hemorrhagic strokes; a diagnostic accuracy study. Am J Emerg Med. 2018;36(11):2064-7.

38. Albert AF, Kirkman MA. Clinical and radiological predictors of malignant middle cerebral artery infarction development and outcomes. J Stroke Cerebrovasc Dis. 2017;26(11):2671-9.

39. Monnin P, Sfameni N, Gianoli A, Ding S. Optimal slice thickness for object detection with longitudinal partial volume effects in computed tomography. J Appl Clin Med Phys. 2017;18(1):251-9.

40. Lee S, Kim YO, Baek JS, Ryu JA. The prognostic value of optic nerve sheath diameter in patients with subarachnoid hemorrhage. Crit Care. 2019;23(1): 65 .

\section{Publisher's Note}

Springer Nature remains neutral with regard to jurisdictional claims in published maps and institutional affiliations.

Ready to submit your research? Choose BMC and benefit from:

- fast, convenient online submission

- thorough peer review by experienced researchers in your field

- rapid publication on acceptance

- support for research data, including large and complex data types

- gold Open Access which fosters wider collaboration and increased citations

- maximum visibility for your research: over $100 \mathrm{M}$ website views per year

At BMC, research is always in progress.

Learn more biomedcentral.com/submissions 